\title{
REAL TIME OPTIMIZATION OF THE STERILIZATION PROCESS IN A CANNING INDUSTRY
}

\author{
Carlos Vilas* \\ (Bio)Process Engineering Group (IIM-CSIC), c/ Eduardo Cabello, 6, Vigo (Spain), carlosvf@iim.csic.es \\ Antonio A. Alonso \\ (Bio)Process Engineering Group (IIM-CSIC), c/ Eduardo Cabello,6, Vigo (Spain), antonio@iim.csic.es
}

\begin{abstract}
Sterilization process is aimed to inactivate potentially harmful microorganisms. To that purpose the packaged food is subject to a time/temperature profile. In the canning industry such profiles are chosen based on the experience of the operator. In the presence of perturbations, such as steam supply problems, operators must react and design new profiles which, in most of the cases, are too conservative and/or may lead to the batch rejection, either because of quality or safety reasons.

In order to overcome this problem, we propose in this work a model based real time optimization (RTO) strategy. The model, which describes the different relevant aspects of the plant (retort/can temperature, color dynamics, microorganism lethality, energy consumption, etc.) is used to predict the behavior of the system. Plant measurements are taken periodically and, in the event of a perturbation, an optimization procedure is run to compute a new time/temperature profile based on the past measurements.
\end{abstract}

Keywords: Mathematical Modeling; Real Time Optimization; Sterilization of Packaged Foods; Food Safety; Food Quality.

\section{INTRODUCTION}

The aim of the sterilization process is to inactivate microorganisms present in the product that might cause health problems upon consumption. Thermal sterilization of solid foodstuff consists of exposing the product to relatively high temperatures (around $110-130{ }^{\circ} \mathrm{C}$ ) during a given amount of time.

Typical sterilization cycles consist of three stages: heating stage where the temperature increases from room temperature to a given set point; maintenance stage where the set point temperature is kept during a given amount of time; and cooling stage aiming at rapidly decreasing product temperature so that cans can be handled and quality can be kept.
Thermal sterilization process has several drawbacks: (i) it is a particularly demanding operation both in terms of energy consumption and process time; (ii) it adversely affects product quality (real or perceived) [11] since nutrients or sensory parameters (color or texture for instance) are deteriorated.

In practice, industry operates using some preestablished profiles to carry out the sterilization. However, sterilization at industry levels is subject to unknown perturbations, such as deficiencies in the steam supply; scheduling problems, etc., that affect the process. Operators react to these perturbations following some conservative recipes to ensure safety of the final product. However, this may have a large impact on the final product quality; energy consumption; or final processing time; leading, in some cases, to the rejection of the batch.

Model based real time optimization RTO $[4,1]$ using current plant measurements allows for designing strategies to correct the presence of unexpected disturbances taking into account their effects on quality, process time and energy consumption.

The aim of this work is to design a RTO strategy able to detect, using plant measurements, when a perturbation leads to food safety issues and, in such a case, compute a new optimal profile able to minimize the effects of such perturbation. The objective function considered combines energy consumption, food quality (understood as surface color) and process time. The relative weight of these criteria can be modified by changing some parameters in the objective function. Decision variables in the optimization block are heating and maintenance time as well as maintenance temperature.

The RTO profiles implemented in this work, consist of constant sterilization profiles due to legislation issues. However, larger improvement might be acquired if temperature set point is allowed to be modified during the sterilization (variable retort temperature, VRT) $[12,5,6,1]$.

The manuscript is structured as follows: first we 
briefly describe the sterilization process carried out in the considered canning plant and illustrate the problems caused by service steam issues. Then we develop the mathematical model describing the dynamics of the plant and we briefly state the RTO problem. Finally, we present the main results of the work.

\section{THE STERILIZATION PROCESS}

In the case considered in this work, superheated water is used to increase the temperature of the food packaged products as shown in Figure 1. Such water is heated using a plate heat exchanger

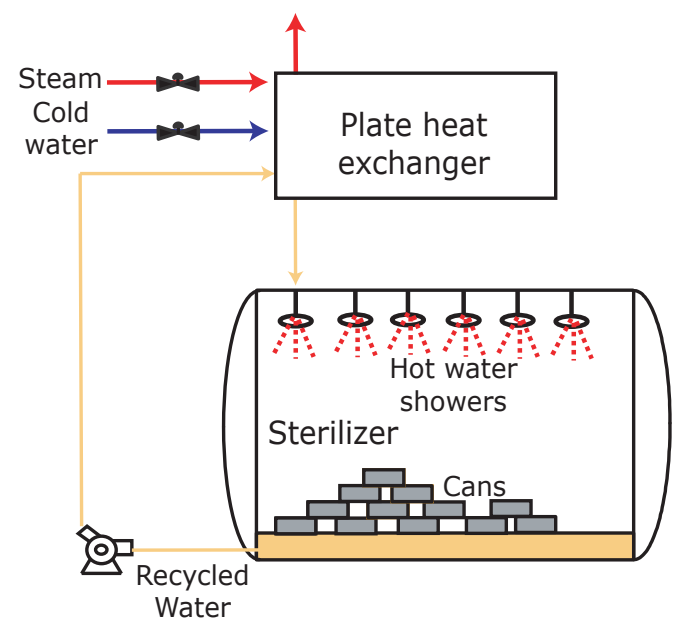

Figure 1: Sterilizer unit with plate heat exchanger.

(PHE). At the beginning of the process preheated water $\left(\approx 60{ }^{\circ} \mathrm{C}\right)$ is introduced into the sterilizer and recycled to the $\mathrm{PHE}$. As the process evolves water temperature in the retort increases until it reaches the operation point (typically a temperature between 110 and $130{ }^{\circ} \mathrm{C}$ ). Such temperature is kept for a given amount of time after which the steam valve in the PHE is closed and cold water is introduced to cool down the cans.

At the end of the process microorganism lethality at the coldest point must be above a given value (typically $8 \mathrm{~min}$ ).

Figure 2 shows different sterilizer temperature profiles (top figure), implemented on an industrial canning plant for RO-80 can types and their associated microbial lethality evolution (bottom figure). Dash horizontal line in the lethality plot indicates the minimum safety requirements. Such profiles correspond to two typical sterilization temperatures: 116 and $121^{\circ} \mathrm{C}$. Several aspects can be highlighted in the figure:

- Cycles carried out at $121{ }^{\circ} \mathrm{C}$ are, obviously shorter.
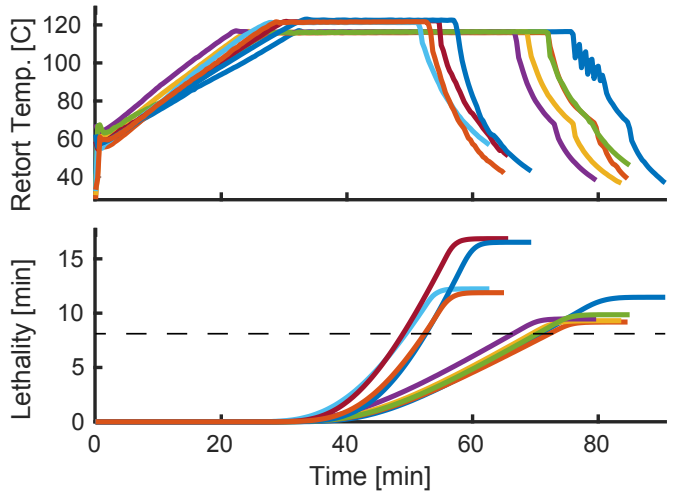

Figure 2: Top figure: sterilizer temperature profiles in real cases carried out at the considered canning plant. Bottom figure: lethality evolution associated with the temperature profiles.

- Even for the same sterilization temperature a large variability (in final time) can be appreciated. This is caused by a large variability in the heating stage time due to: (i) different number of sterilizers connected to the PHE what makes service pressure to drop; or (ii) different number of can loaded in the sterilizers.

- The variability in the heating stage times forces the operator to make decisions on the final cycle time in order to ensure safety and avoid the rejection of the batch.

- In general, the new cycle times are conservative (lethality larger than required) causing quality loses and increasing, unnecessarily, process time.

- A large variability in the lethality reached at end of the process

Model based real time optimization can be used to minimize the effects of plant perturbations ensuring safety and minimizing quality losses, energy consumption or process time.

\section{MATHEMATICAL MODEL}

In this section we present the equations describing: (i) the PHE and sterilizer temperature dynamics; (ii) the packaged food product temperature evolution and distribution; and (ii) the effect of food temperature on safety and quality.

\subsection{PLATE HEAT EXCHANGER}

A PHE consists of a number of plates through which cold and hot fluids circulate (see Figure 3). In the canning plant under study, the hot fluid stream enters the PHE from the top side. The 


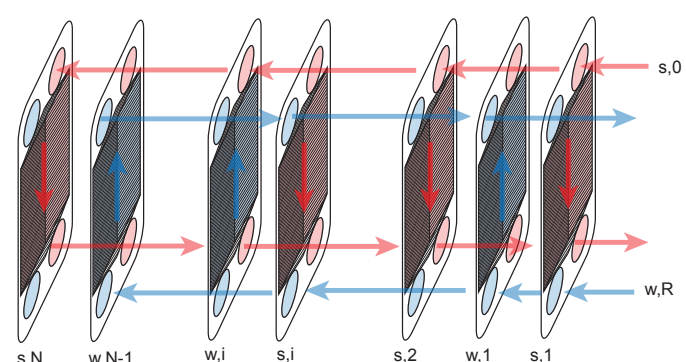

Figure 3: Sterilizer unit with plate heat exchanger.

system allows the entrance of hot fluid every other plate. Once the fluid reaches the end of the PHE it returns through a pipe located at the bottom of the system. The remaining plates correspond to the cold fluid which enters through the bottom and comes out through the top. Cold and hot fluids correspond to water and saturated steam, respectively.

PHE temperature dynamics are obtained through an energy balance in each of the plates. Some assumptions are made:

- "Cold" water inlet stream temperature is the same for all the plates.

- Steam inlet stream temperature is the same for all the plates.

- The plate heat exchanger is well isolated, i.e. there are no heat loses.

- No phase change in the steam or water streams occurs.

- Fluid flow rate in all "cold" water plates is the same.

- Fluid flow rate in all steam plates is the same.

For the sake of clarity, only the final set of partial differential equations are indicated here.

Equations for the plates containing cold water are of the form

$$
\begin{gathered}
m_{w} c_{p, w} \frac{\partial T_{w, i}}{\partial t}=-v_{w} m_{w} c_{p, w} \frac{\partial T_{w, i}}{\partial z}+ \\
U A_{p}\left(T_{s, i}-2 T_{w, i}+T_{w, i+1}\right)
\end{gathered}
$$

with $m_{w}, v_{w}$ and $T_{w, i}$ being, respectively, the mass, velocity and temperature of water in each of the plates. $c_{p, w}$ is the specific heat of water whereas $U$ and $A_{p}$ are the heat transfer coefficient and the contact area between plates. $T_{s, i}$ corresponds with the temperature of steam in each of the plates, which is computed by solving the following set of partial differential equations:

$$
m_{s, 1} c_{p, s, 1} \frac{\partial T_{s, 1}}{\partial t}=-v_{s, 1} m_{s, 1} c_{p, s, 1} \frac{\partial T_{s, 1}}{\partial z}+
$$

$$
\begin{gathered}
U A\left(T_{w, 1}-T_{s, 1}\right) \\
m_{s, i} c_{p, s, i} \frac{\partial T_{s, i}}{\partial t}=-v_{s, i} m_{s, i} c_{p, s, i} \frac{\partial T_{s, i}}{\partial z}+ \\
U A\left(T_{w, i-1}-2 T_{s, i}+T_{w, i}\right) \\
m_{s, N} c_{p, s, N} \frac{\partial T_{s, N}}{\partial t}=-v_{s, N} m_{s, N} c_{p, s, N} \frac{\partial T_{s, N}}{\partial z}+ \\
U A\left(T_{w, N-1}-T_{s, N}\right)
\end{gathered}
$$

where $m_{s, i}, v_{s, i}$, and $c_{p, s, i}$ are, respectively, the mass, velocity, and specific heat of steam in each of the plates. In this case, model parameters as well as fluid flow characteristic allow us to approximate the convection term by a finite differences scheme with two terms, i.e.:

$$
\frac{\partial T_{s, i}}{\partial z}=\frac{T_{s, i}-T_{s, 0}}{L_{p}} ; \quad \frac{\partial T_{w, i}}{\partial z}=\frac{T_{w, i}-T_{w, R}}{L_{p}}
$$

with $L_{p}$ being the plate height. $T_{s, 0}$ corresponds with the steam service temperature whereas $T_{w, R}$ is the recycle water temperature which is assumed to be the same as the temperature of the water in the sterilizer.

\subsection{THE STERILIZER}

\subsubsection{The sterilizer unit}

A simple energy balance allows us obtaining the equation describing the evolution of the sterilizer water temperature.

$$
\begin{gathered}
m_{w, R} c_{p, w} \frac{\mathrm{d} T_{w, R}}{\mathrm{~d} t}=q_{w} c_{p, w}\left(T_{w, P H E}-T_{w, R}\right)- \\
Q_{c a n}-Q_{a m b}
\end{gathered}
$$

where $T_{w, P H E}$ is the water temperature at the output of the PHE. $Q_{c a n}$ is the heat absorbed by the cans, whose form will be presented in the following section. Sterilizers heat loses are computed as:

$$
Q_{a m b}=h_{c} A_{c}\left(T_{w, R}-T_{a m b}\right)
$$

where $T_{a m b}, h_{c}$ and $A_{c}$ are the surrounding media temperature, heat transfer coefficient between the sterilizer and the surrounding media and the area of the metal cover of the retort.

In order to maintain the set point temperature a PID controller is installed in the canning plant considered. Such controller regulates the sterilizer temperature by modifying the entrance of steam in the PHE. In this regard, steam valve opening is computed as:

$$
\begin{gathered}
u_{s, k}=u_{s, k-1}+K_{p}\left(e_{k}-e_{k-1}\right)+K_{i} \Delta t e_{k}+ \\
\frac{K_{d}}{\Delta t}\left(e_{k}-2 e_{k-1}+e_{k-2}\right)
\end{gathered}
$$

where $\Delta t$ is the time between two consecutive measurements and $e_{k}=T_{w, R}\left(t_{k}\right)-T_{w, R}^{s p}$, with $T_{w, R}^{s p}$ being the set point temperature. 


\subsubsection{Energy consumption}

In order to compute the energy consumption we assume that heat can recovered at the output of the hot stream in the PHE. In this regard, heat consumption is computed as the amount of heat transmitted from the steam to the water in the PHE. Mathematically this can be expressed as:

$$
E_{c}=\int_{0}^{t_{f}} q_{w} c_{p, w}\left(T_{w, P H E}(t)-T_{w, R}(t)\right) \mathrm{d} t
$$

\subsubsection{Packaged food product}

The solid product to be sterilized is packed in cylindrical RO-80 containers, although the procedure would be the same for other type of packages. We assume that the temperature of the retort is the same in all the can surface. Under these conditions symmetry properties can be used to transform the original $3 \mathrm{D}$ problem into a $2 \mathrm{D}$ equivalent one (see Figure 4). As the package product is

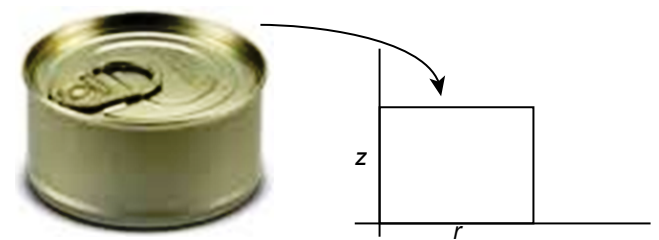

Figure 4: Equivalence between the 3D and 2D spatial domains.

mostly solid, temperature distribution obeys the so-called Fourier equation for heat conduction in cylindrical coordinates, i.e.:

$$
\rho_{p} c_{p, p} \frac{\partial T_{p}}{\partial t}=\kappa\left[\frac{\partial^{2} T_{p}}{\partial z^{2}}+\frac{1}{r} \frac{\partial}{\partial r}\left(r \frac{\partial T_{p}}{\partial r}\right)\right]
$$

where $r$ and $z$ are the cylindrical coordinates representing radius and length respectively. $T_{p}(r, z, t)$ stands for product temperature, whereas $\rho_{p}, c_{p, p}$ and $\kappa$ corresponded to product density, specific heat and thermal conductivity, respectively. The equation is completed with (Robin-type) boundary conditions of the form:

$$
\begin{gathered}
\kappa\left(\frac{\partial T_{p}}{\partial z}\right)_{z=L}=h_{t}\left(T_{R}-\left.T_{p}\right|_{z=L}\right) \\
\kappa\left(\frac{\partial T_{p}}{\partial z}\right)_{z=0}=h_{b}\left(T_{R}-\left.T_{p}\right|_{z=0}\right) \\
\kappa\left(\frac{\partial T_{p}}{\partial r}\right)_{r=R}=h_{r}\left(T_{R}-\left.T_{p}\right|_{r=R}\right) \\
\left(\frac{\partial T_{p}}{\partial r}\right)_{r=0}=0
\end{gathered}
$$

where $h_{x}$, with $x=t, b, r$ corresponds to the convective heat transfer coefficient between the product (top, bottom and right) boundaries and the water from the showers.
We assume that all can in the sterilizer absorb the same amount of heat which is, then, computed as [15]:

$$
Q_{c a n}=n_{c} m_{c} c_{p, c} \frac{\mathrm{d} T_{c, n}}{\mathrm{~d} t}
$$

where

$$
T_{c, n}=\frac{\int_{V} T_{p}(r, z, t) \mathrm{d} V}{V}
$$

with $V$ being the volume of the can.

\subsection{SAFETY AND QUALITY INDICATORS}

Lethality at the cold point $F_{0}$ is considered as the safety indicator. Time evolution of this variable is described by a Thermal Death Time (TDT) kinetic equation of the form [3]:

$$
\frac{\mathrm{d} F_{0}}{\mathrm{~d} t}=10^{\frac{T_{c}(t)-T_{M, r e f}}{z_{M, r e f}}}
$$

Parameters $T_{M, \text { ref }}$ and $z_{M, \text { ref }}$ correspond to Clostridium botulinum bacteria [2] since, in spore form, it is one of the most heat resistant bacteria.

TDT kinetics has been previously used to describe surface color evolution $\left(C_{s}\right)$ (quality parameter) [2]. However, some experimental work showed that, in this case, first order kinetics are more accurate, i.e.:

$$
\frac{\mathrm{d} C_{s}}{\mathrm{~d} t}=-k_{A r r} y
$$

with

$$
k_{A r r}=k_{\text {ref }} \exp \left[\frac{E_{a}}{R}\left(\frac{1}{T_{s}}-\frac{1}{T_{r e f}}\right)\right]
$$

where $k_{r e f}$ is a reference constant at $T_{r e f} . E_{a}$ and $R$ denote, respectively the activation energy and universal gas constant. $T_{s}$ is the temperature at the surface of the food product.

Unknown model parameters were estimated following the criteria in [14].

\section{STERILIZATION PROCESS REAL TIME OPTIMIZATION}

The scheme for real time optimization used in this work is schematically presented in Figure 5. First, a given set point for sterilizer temperature and process time is selected. The PID controller computes the steam valve openings $\left(u_{s}\right)$ to reach and maintain such temperature. Actual plant measurements (sterilizer temperature, $T_{w, R}$ ) are fed to the model which predicts the final lethality of the products accordingly. If lethality fulfills safety requirements, the profile is kept otherwise a new optimal profile is computed by means of an optimization step taking into account available plant measurements. 


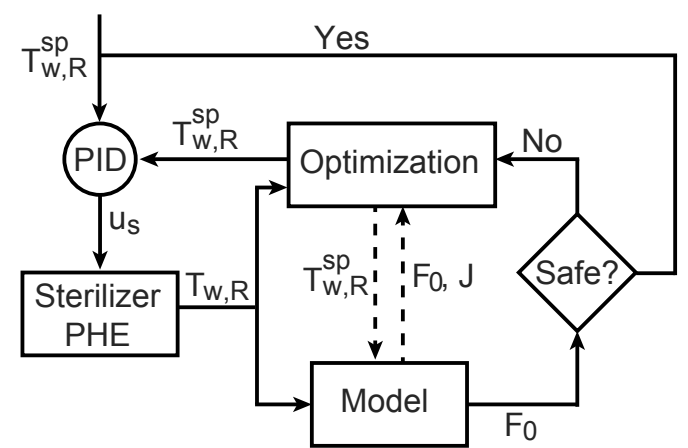

Figure 5: Flow chart of the RTO scheme proposed.

In practice, options for optimization are limited by law. New profiles, as for instance those using variable retort temperature (VRT), must be validated by food control agencies before they can be implemented. Therefore, in this work, we will focus on constant temperature profiles.

The optimization block will search for the control profile, i.e. heating time and temperature of the maintenance stage $\left(t_{h}, T_{w, R}^{s p}\right)$, that minimizes a given objective function $(J)$. In this work, $J$ combines three variables: energy, surface color and process time:

$$
J_{t_{h}, T_{w, R}^{s p}}=w_{1} E_{c}+w_{2}\left(100-C\left(t_{f}\right)\right)+w_{3} t_{f}
$$

where $w_{1}, w_{2}$ and $w_{3}$ are given weights which may change depending on the primary objective of the operator (saving energy, final product quality or saving process time).

The minimization of $J$ is carried out taking into account the following constraints on the model variables and bounds on the decision variables:

1. $F_{0}\left(t_{f}\right)>8 \mathrm{~min}$.

2. Model equations (1)-(14).

3. $110{ }^{\circ} \mathrm{C} \leq T_{w, R}^{s p} \leq 130{ }^{\circ} \mathrm{C}$.

4. $0 \min \leq t_{h} \leq 80 \min$

where $t_{f}$ and $t_{h}$ are, respectively, final process time and heating stage time.

In order to find the solution of this problem, a hybrid stochastic-deterministic method based on the scatter search approach will be employed. In particular, the algorithm eSS (Enhanced Scatter Search) [7] will be used. Note that the dynamics of the sterilizer and PHE are explicitly considered therefore, the resulting profiles will be, by construction feasible.

Other type of objective functions and constraints could be also considered in this methodology. In this regard, nutrient retention at the end of the process, personnel costs, etc. could be taken into account. On the other hand, quality could be considered as a constraint instead of a variable to be maximized.

Plant model mismatch is not considered in this work. However, the methodology described in [10] could be applied to such purpose.

\section{RESULTS AND DISCUSSION}

In order to illustrate the predictive capabilities of the model, we have chosen a particular experiment among those presented in Figure 2. Figure 6 shows the comparison between the data corresponding to such experiment (marks) and the model simulation results (continuous lines). Blue colors refer to retort temperature whereas red colors correspond to lethality. In this regard, it should be pointed out that lethality cannot be directly measured. Red marks were obtained through Eqn (13) where $T_{c}$ corresponds to temperature measurements at the center of the can. As shown in the figure, the

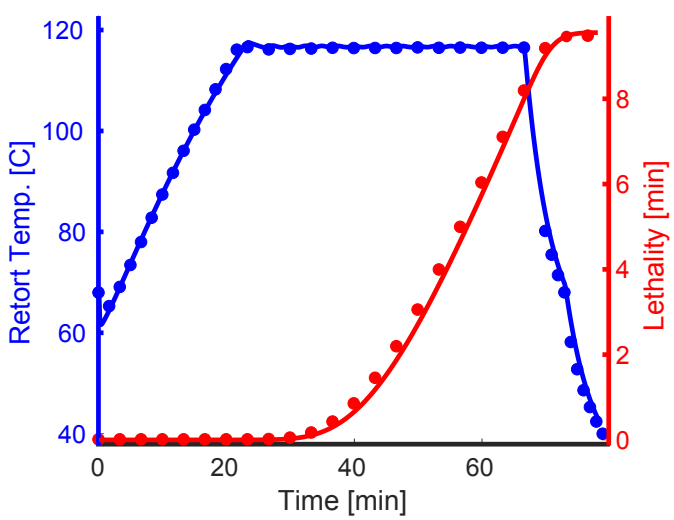

Figure 6: Comparison between experimental data (marks) obtaining at a canning industry and model simulation results (continuous lines).

model is able to reproduce the experimental data with a satisfactory degree of accuracy.

Once the model has been tested, it can be used for optimization purposes. Three approaches are followed. In the first one, sterilization temperature is assumed to be the same as the one used in the experiment so the decision variable is the sterilization time. In the other two, we also included the sterilization temperature as a decision variable. The differences between these two approaches are the weights in the objective function. In the first one more importance is given to quality whereas in the second one more importance is given to energy saving. The results are summarized in Figure 7 and Table 1.

In the process originally implemented in the canning plant (blue line), steam pressure was lower 
than expected causing the heating stage to be longer than predicted. In order to ensure safety, the operator decided to extend the maintenance stage with respect the original plan. The value of
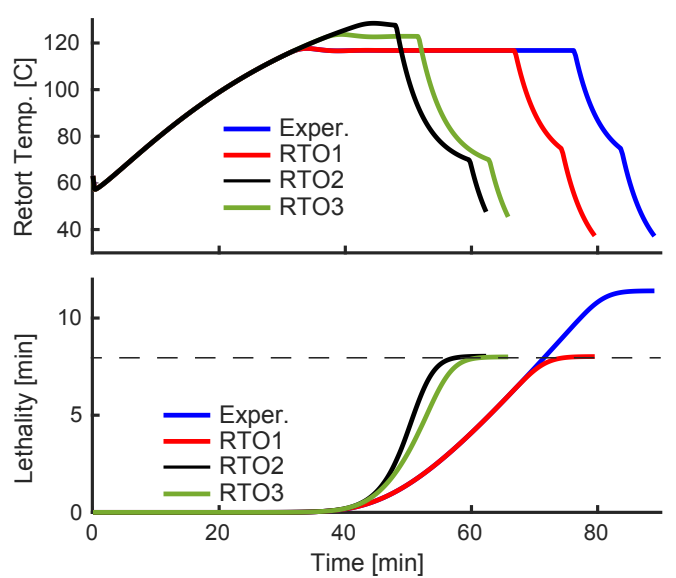

Figure 7: Comparison between a experiment carried out in a canning plant and three RTO profiles. Top figure: retort temperature. Bottom figure: lethality evolution. Horizontal dashed line indicates the lethality constraint.

lethality reached at the end was 11.5 min and the duration of the cycle was 89 min. The product was over-processed.

The RTO approach allowed detecting the steam service problem so new profiles were computed. As shown in the Figure 7, in all RTO profiles final lethality was $8 \mathrm{~min}$, i.e the value of the constraint defined in the optimization problem. The

Table 1: Comparison between the optimization schemes. Percentages of improvement are given with respect the profile originally implemented in the canning plant. Negative values indicate worser performance with respect such profile.

\begin{tabular}{llll}
\hline & \multicolumn{3}{l}{ Percentage of improvement } \\
\cline { 2 - 4 } & Energy & Color & Time \\
\hline RTO1 & 2.7 & 2.8 & 10.7 \\
RTO2 & -5.7 & 8.0 & 30.0 \\
RTO3 & 1.3 & 5.7 & 21.1 \\
\hline
\end{tabular}

first RTO profile (red line), improved the original profile in all senses. It allows reducing process time in around 10 min saving energy and improving surface color (see Table 1). The second RTO profile (black line) is the most aggressive, maintenance temperature was $127.6{ }^{\circ} \mathrm{C}$. This profile was, evidently, the shortest one (around $27 \mathrm{~min}$ shorter than the original profile). It was also able to increase quality (color) by a $8.0 \%$ although it is the most energy consuming profile. Changing the weights in the objective function -Eqn. (15)- to give more importance to energy saving, resulted into the third RTO profile (green line). This profile, with a maintenance temperature of $120.2^{\circ} \mathrm{C}$, also improves the process in all senses allowing to reduce cycle time in around $19 \mathrm{~min}$.

Disturbances during the maintenance stage are more unusual, however in such events the RTO scheme would also react and compute new profiles preventing the over-processing of the product.

Finally, as mentioned above, the RTO profiles implemented in this work, considered constant sterilization profiles. However, larger improvement might be acquired using VRT. In such cases, the optimal control problem can be approached by using the control vector parametrization (CVP) method in which the solution is approximated by piece-wise low order polynomials (see $[9,13,8]$ for details). This allows converting the original problem into a nonlinear programming problem (NLP) that may be solved using a global optimizer.

\section{CONCLUSION}

In this work we presented a methodology for minimizing the effects of unexpected disturbances during the sterilization process of a canning industry. We showed that in many cases product is overprocessed as a result of conservative adjustments made to counteract such disturbances and ensure food safety.

The methodology consisted of using plant measurements and a mathematical model of the plant to detect when a perturbation will cause food safety problems. In such event, an optimization stage is carried out (using plant measurements as an input) to recompute sterilization temperature and time. This allowed us minimizing the effects of the disturbance on food quality, energy consumption and process time.

Finally, we illustrated the benefits of such scheme with respect the classical procedure.

\section{Acknowledgments}

These results are part of the CoPro project which has received funding from the European Union's Horizon 2020 research and innovation programme under grant agreement No 723575 .

\section{Referencias}

[1] A. A. Alonso, A. Arias-Méndez, E. BalsaCanto, M. R. García, J. I. Molina, C. Vilas, and M. Villafín. Real time optimization for quality control of batch thermal sterilization of prepackaged foods. Food Control, 32(2):392-403, 2013. 
[2] J. R. Banga, A. A. Alonso, J. Gallardo, and R. I. Pérez-Martín. Kinetics of thermal degradation of thiamine and surface colour in canned tuna. $Z$ Lebensm Unters Forsch, 197(2):127-131, 1993.

[3] A. Casp and J. Abril. Procesos de conservación de alimentos. Tecnología de alimentos. Mundi-Prensa, 2003.

[4] M. Diehl, H. G. Bock, J. P. Schloder, R. Findeisen, Z. Nagy, and F. Allgower. Real-time optimization and nonlinear model predictive control of processes governed by differentialalgebraic equations. Journal of Process Control, 12(2):577-585, 2002.

[5] T. D. Durance. Improving canned food quality with variable retort temperature processes. Trends in Food Science and Technology, 8(4):113-118, 1997.

[6] T. D. Durance, J. L. Dou, and J. Mazza. Selection of variable retort temperature processes for canned salmon. Journal of Food Process Engineering, 20(1):65-76, 1997.

[7] J. A. Egea, E. Vazquez, J. R. Banga, and R. Marti. Improved scatter search for the global optimization of computationally expensive dynamic models. Journal of Global Optimization, 43(2-3):175-190, 2009.

[8] F. Fournier, M. Fikar, and M. A. Latifi. Control vector parametrisation in optimal control of a batch electrochemical reactor. Chemical and Biochemical Engineering Quarterly, 12(2):81-85, 1998.

[9] C. J. Goh and K. L. Teo. Control parameterization: a unified approach to optimal control problems with general constraints. Automatica, 24:3-18, 1988.

[10] T. Rodríguez-Blanco, D. Sarabia, J. L. Pitarch, and C. de Prada. Modifier adaptation methodology based on transient and static measurements for rto to cope with structural uncertainty. Computers \& Chemical Engineering, 106:480-500, 2017.

[11] A. A. Teixeira and G. S. Tucker. On-line retort control in thermal sterilization of canned foods. Food Control, 8:13-20, 1997.

[12] A. A. Teixeira, G. E. Zinsmeister, and J. W. Zahradnik. Computer simulation of variable retort control and container geometry as a possible means of improving thiamine retention in thermally processed foods. Journal of Food Science, 40(4):656-659, 1975.
[13] V. S. Vassiliadis, R. W. H. Sargent, and C. C. Pantelides. Soluion of a class of multistage dynamic optimization problems 2 . problems with path constraints. Industrial \& Engineering Chemistry Research, 33(9):21232133, 1994.

[14] C. Vilas, A. Arias-Méndez, M. R. García, A. A. Alonso, and E. Balsa-Canto. Towards predictive food process models: A protocol for parameter estimation. Critical Reviews in Food Science and Nutrition, 58(3):463-449, 2018.

[15] C. Vilas, M. R. García, J. R. Banga, and A. A. Alonso. Desarrollo de una librería de componentes en EcosimPro para la operación de plantas de procesamiento térmico de alimentos. Revista Iberoamericana de Automática e Informática industrial, 5(1):51-65, 2008.

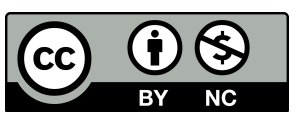

(C) 2018 by the authors. Submitted for possible open access publication under the terms and conditions of the Creative Commons Attribution CC-BY-NC 3.0 license (http://creativecommons.org/licenses/by-nc/3.0/). 